\title{
A Randomized Controlled Trial of Awareness Training Program (ATP), a Group-Based Mahayana Buddhist Intervention
}

\author{
Bonnie Wai Yan $\mathrm{Wu}^{1} \cdot$ Junling Gao ${ }^{1} \cdot$ Hang Kin Leung ${ }^{1} \cdot$ Hin Hung Sik ${ }^{1}$
}

Published online: 17 January 2019

(C) The Author(s) 2019

\begin{abstract}
Objectives This study evaluated the effectiveness of a Mahayana Buddhist teaching- and group-based intervention, the Awareness Training Program (ATP), which is textually aligned to a Mahayana Sütra so that its theory and practice are coherent. The ATP aims to alleviate stress by enhancing participant's compassion and wisdom of nonattachment.

Methods Middle-aged working adults $(n=122)$ in Hong Kong participated in this randomized waiting-list controlled trial. Selfreported psychological questionnaires were used to assess the participants' level of stress (PSS), sense of coherence (SOC), psychological well-being (GHQ), and nonattachment (NAS) at pretest, posttest, and 3 months later.

Results The data showed significant improvements in the intervention group over the controls for all outcome measures at posttest and 3 months later (post and post-3 PSS: $\eta p ~ 2=.15$ and .16 ; post and post- 3 SOC: $\eta p$ 3 GHQ: $\eta p ~ 2=.16$ and .13; post and post-3 NAS: $\eta p 2=.17$ and .14). A mediation analysis demonstrated that nonattachment mediated both the treatment and the maintenance effects for all outcome variables (post and post-3 PSS: indirect effect ab $=-$ 1.50 and -1.70 ; post and post-3 SOC: $a b=2.48$ and 3.61; post and post-3 GHQ: $a b=-1.65$ and -1.56 ; bootstrap $95 \%$ confidence interval (CI), CI does not include zero).

Conclusions The ATP may effectively reduce stress and improve a sense of coherence and psychological well-being by enhancing participants' wisdom of nonattachment. This study provides promising evidence that the ATP could be an effective intervention for middle-aged working adults.
\end{abstract}

Keywords Awareness Training Program $\cdot$ Mahayana Buddhism $\cdot$ Meditation $\cdot$ Stress $\cdot$ Nonattachment .

Wisdom of nonattachment $\cdot$ Compassion

Over the past few decades, there has been increasing interest in incorporating Buddhist practices into group-based interventions for treating various psychological problems in the behavioral, social, and health sciences. The Buddhist practice of mindfulness has been incorporated into many of these groupbased interventions as a key component. Such practices are generally known as "mindfulness-based interventions" (MBIs). Scholars have demonstrated the efficacy and effectiveness of many of these MBIs in improving participants' psychological and physical health.

However, some scholars have shown that many MBIs vary in the type of mindfulness practice and in the manner that is incorporated into the interventions (Chiesa and Malinowski 2011; Singh et al. 2008; Van Gordon et al. 2015). Many

Hin Hung Sik

hinhung@hku.hk

1 Buddhism and Science Research Lab, Centre of Buddhist Studies, The University of Hong Kong, Hong Kong, Hong Kong scholars have also noted that one possible issue with these MBIs is that they may have applied mindfulness practice in an out-of-context manner (Bodhi 2011b; Shonin et al. 2013b; Singh et al. 2008) that "divorces it from its profoundly transformative potential" (Kabat-Zinn 2015).

Another possible issue with these interventions is that they may have incorporated components from various Buddhist models of the elimination of suffering without considering if they are coherent. As Bodhi (2011a) has noted, there is a danger in creating "cognitive dissonance" by merging theories and/or techniques from different schools and/or traditions. The reason for this argument is that each individual school and/or tradition has its own theoretical model and assumptions, which guide and dictate the development of the corresponding system of practice. Nevertheless, Shonin and Van Gordon (2015) have a different perspective on this discussion of lineages and traditions. They argued that "the Buddha wasn't concerned with endorsements or lineages, he was only interested in whether a meditation teacher had tamed their mind" (Shonin and Van Gordon 2015, p. 143) and "lineage is a means of guiding 
people to enlightenment, but it is not enlightenment itself" (Shonin and Van Gordon 2015, p. 143). Therefore, it is argued that authentic Dharma lineages and traditions must be open to evolution and not become attached to whatever guiding principles they deem define them.

Despite these differing views, some scholars still believe that building an intervention that systematically incorporates a coherent Buddhist model of the elimination of suffering into its theoretical model and program content is a logical development given that many of these MBIs have been shown to be efficacious by incorporating only part of the Buddhist system of eliminating suffering (Sik 2010; Teasdale et al. 2003; Van Gordon et al. 2014). According to Van Gordon et al. (2015), these MBIs could be regarded as "second-generation MBIs," and when compared to first-generation MBIs, such as Mindfulness-Based Stress Reduction (MBSR), these "second-generation MBIs, such as Meditation Awareness Training (MAT), have integrated a greater range of meditation techniques and subscribe to a definition of mindfulness that is arguable to be more congruent with the traditional Buddhist construction" (Van Gordon et al. 2016, p. 364).

This could be further developed by formulating an intervention that systematically combines a Buddhist theoretical model with a compatible meditation practice. Recent examples of second-generation MBIs include Mindfulness-Based Positive Behavior Support (Singh et al. 2013, 2014, 2018) which includes the four immeasurables, compassion, attachment, and the five hindrances, and explicit teachings on Buddhist ethics, and Mindfulness-Based Symptom Management (Monteiro et al. 2018) which includes the Five Skilful Habits - the five lay Buddhist precepts - presented as ethical themes. However, current second-generation MBIs do not explicitly include Mahayana Buddhist teachings or groupbased interventions that adopt a textually aligned approach, and that has a coherent theory and praxis.

"Mahayana Buddhist teaching," "textually aligned," and "coherent theory and praxis" are important components to consider when formulating an MBI for the following reason: Mahayana Buddhism, unlike the more classical Theravada Buddhism that focuses on eliminating suffering, emphasizes the cultivation of compassion and wisdom to develop spiritual advancement, which will eventually lead to the attainment of Buddhahood. In a way, Mahayana Buddhism's emphasis of the cultivation of compassion and wisdom follows a "wellness model," whereas Theravada Buddhism's focus on the elimination of suffering is more consistent with a "medical model."

The importance of being "textually aligned" concerns tracing back to the source of the teachings, thereby avoiding the potential danger of inconsistency between theory and praxis. There are thousands of sacred Buddhist texts that belong to many different schools and lineages, and each has its own emphasis and theoretical inclination. Further complicating the situation is that within both Theravada and Mahayana
Buddhism, there are subschools and lineages that each has a unique theory and praxis. Therefore, to be "textually aligned" is to ensure that the theory and praxis that are employed by Buddhist-inspired interventions are derived from text(s) that have a similar background in both theory and praxis and, as a result, avoid the possibility of creating "cognitive dissonance."

The theoretical foundation of the newly developed Awareness Training Program (ATP) is rooted in the Mahayana Buddhist text of the Sandhinirmochana Sütra (Keenan 2000), which is one of the most important texts of the Yogācāra tradition. The main philosophy of the Yogācāra tradition is the concept of "consciousness-only" or "mind-only." The tradition asserts that "the world as it appears to the unenlightened one is a mere representation of the consciousness" (Kochumuttom 1999, p. 5), which is similar to "seeing something by means of a clean mirror with the object before it, thinking one is seeing an image, and thinking there is a separate image apart from the object" (Cleary 1995, p. 45). As with other Buddhist schools, Yogācāra agrees that the source of human suffering is the result of "ontological addiction," which refers to the unwillingness to relinquish one's attachment to an erroneous and deep-rooted belief in an inherently existing "self" or "I," along with the "impaired functionality" that arises from such a belief (Shonin et al. 2013a). The Sandhinirmochana Sütra states that the cause of this delusion and attachment is that "people add a conceptualized nature on top of the dependent nature and the real nature" (Cleary 1995, p. 30).

To help people overcome this delusion and attachment, the Sandhinirmochana Sütra promises to expound a teaching that is "completely explicit" and requires no interpretation to be understood (Willams 2004, p. 79). Instead of merely saying that all things are inherently empty to help people to let go of their ontological addiction to the concept of self, the Sütra taught that on the one hand, all our phenomenological experiences can be explained and qualified by one of three types of characteristics (三自性); on the other hand, these three types of characteristics, when examined closely, will reveal three types of emptiness or essencelessness characteristics (三種無自性 性). These three types of characteristics are the following: (1) the characteristic of mere conceptual grasping (遍計所執 性) or the patterns that are entirely imagined or superficially established, for example, the name of a flower such as "rose"; (2) the characteristic of dependent origination (依他起性) or the patterns or forms that "come to be" depending on the coming together of other conditions, for example, a flower, which comes into being based on the coming together of its components such as petals, a stem and fragrance; and (3) the perfect characteristic of reality (圓成實性) or the full perfection characteristic that can be experienced by a fully enlightened person and of which such a person can be conscious, for example, the emptiness nature of a flower (Cleary 1995, pp. 23-24). The three types of emptiness or essencelessness 
characteristics are (1) the essencelessness of characteristics (相 無自性性), (2) the essencelessness of birth (生無自性性), and (3) ultimate essencelessness (勝義無自性性), which are, in a way, the other side of the coin of the three types of characteristics, namely, their intrinsic emptiness natures. For example, "rose," a superficially established name, can carry much emotional value. However, when examined carefully, its ultimate characteristic is essencelessness, and it is intrinsically empty of essence because it is only a subjective concept that exists in a person's mind. Therefore, by understanding the three types of essencelessness characteristics or their intrinsic emptiness natures, a person can gain wisdom and insight to help him/her to forgo the attachment to all phenomenological experiences that would fall within the three types of characteristics and to therefore develop the wisdom of nonattachment.

To complement the teaching of the three types of characteristics and the three types of essencelessness characteristics, the Sandhinirmochana Sütra also includes a corresponding meditation method. According to Cleary (1995, p. vii), one of the translators of the Sütra, this meditation method "was the classical way, and many of the shortcomings and aberrations of modern Western meditation cults can be traced to abandonment of this tradition."

Similar to other traditional Buddhist schools, the Sandhinirmochana Sütra divides the practice of meditation into two parts: tranquility (śamatha) and observation (vipaśyanā). The Buddha began the teaching of meditation in the Sütra with a question from Maitreya, who asked based on what and dependent on what does a Mahayana practitioner practice meditation? The Buddha answered that it is based on the texts of the Mahayana teachings and is dependent on the commitment to attain perfect enlightenment (bodhicitta) (Keenan 2000, p. 51). The Sütra delineates that to develop wisdom and eliminate delusion, it is necessary to follow the progressive pedagogical steps to develop wisdom, which are (1) wisdom realized by learning (śrutamay $\bar{\imath}$ prajñā) (聞所成 慧), (2) wisdom realized by thinking (cintāmaȳ prajñā) (思 所成慧), and (3) wisdom realized by practice (bhāvanāmaȳ prajñā) (修所成慧) (Keenan 2000, p. 63). These steps are also known as the Buddhist pedagogy of the three wisdoms or "the three kinds of knowing."

Practitioners who use this method of meditation focus on the teaching of the scriptures, and they gather and summarize them into a collective whole (for example, a poem or a mantra) to attentively contemplate them (Keenan 2000, p. 56). The ultimate aim of this practice is to enable the practitioner to see and fully understand that all the images and concepts upon which one has focused during either one's meditative practices or one's experiences in daily life are only images and concepts that are created by one's own consciousness, and ultimately, "there is nothing at all seeing anything at all" (Cleary 1995, p. 45 ). By seeing the emptiness nature of phenomena or the three types of essencelessness, a practitioner will eventually eliminate delusion and attachment, which are the root causes of suffering. This is why we theorize that the development of wisdom in the form of nonattachment would be the key to the entire ATP, the mediating factor to facilitate the reduction of delusion, stress, and suffering. It was hypothesized that compared to the control group, the middle-aged working people who receive ATP would demonstrate significant improvements in the areas of stress reduction, stress management, psychological well-being and nonattachment and that these improvements could be maintained at a 3-month follow-up.

\section{Method}

\section{Participants}

The participants were recruited through radio and universitywide promotion. The inclusion criteria for the participants include people (i) who are aged 30 to 60 years, (ii) who are working adults, (iii) who had achieved an educational level of upper secondary school or above, and (iv) who self-reported at least moderate distress with a score $\geq 4$ (out of 7 ) in one of the following aspects: work, family, love affairs, interpersonal relationships, finances, health, or social environment. The exclusion criteria include people (i) who have been diagnosed with psychosis or mental illness, (ii) who are currently engaged in psychotherapy, and (iii) who have previously taken up intensive meditation training or practice. Participation in this study was on a voluntary basis and was not rewarded.

Seventy participants were required to detect a difference of means in a $2 \times 3$ mixed design analysis of variance (ANOVA) for a small effect size $(d)$ of 0.4 , an alpha of 0.05 , and $80 \%$ power by using the Gpower software (http://www.gpower.hhu. de/) to conduct the statistical power analysis (Franz et al. 2007, 2009). In addition, a $20 \%$ attrition rate was assumed. Thus, a total sample size of 88 participants would be required. Eventually, 122 participants were recruited and randomly assigned to either the ATP group or a waitlist control group. More participants were recruited than the calculated sample size because we also considered group-size efficiency, costs, and logistical arrangements. Both the intervention group and the control group completed the self-reported psychological questionnaires at baseline, post-intervention, and 3 months after intervention. The demographic and baseline characteristics of each allocation condition are presented in Table 1 . The potential confounding variables were examined before the evaluation of the hypotheses. The results showed that the participants in the two groups did not differ in their demographic characteristics (i.e., gender, age, education level, marital status, and religious belief) and mean scores of the participants' level of stress (PSS) $(t(120)=1.39, p=.17)$, sense of coherence $(\mathrm{SOC})(t(120)=-$ $0.53, p=.60)$, psychological well-being $(\mathrm{GHQ})(t(120)=0.36$, $p=.72)$, and nonattachment (NAS) $(t(120)=-.90, p=.37)$ at 
Table 1 RCT participants' characteristics in mean, standard deviation, frequency and percentage, and independent $t$ tests/chi-square comparisons between the intervention and the control groups at baseline

\begin{tabular}{|c|c|c|c|c|}
\hline Variables & Subgroups & $\operatorname{ATP}(n=61)$ & Control $(n=61)$ & Test statistic \\
\hline Age & Mean (SD) & $46.33(8.35)$ & $46.82(7.95)$ & $t(120)=-.33, p=.74$ \\
\hline Gender $[n(\%)]$ & Female & $43(70.5)$ & $44(72.1)$ & $\chi^{2}(1)=.040, p=.84$ \\
\hline \multirow[t]{4}{*}{ Education level $[n(\%)]$} & Upper secondary & $12(19.7)$ & $12(19.7)$ & \multirow[t]{4}{*}{$\chi^{2}(3)=0.18, p=.98$} \\
\hline & Post-secondary/university (nondegree) & $11(18.0)$ & $10(16.4)$ & \\
\hline & Post-secondary/university (degree) & $19(31.1)$ & $18(29.5)$ & \\
\hline & Postgraduate & $19(31.1)$ & $21(34.4)$ & \\
\hline \multirow[t]{3}{*}{ Marital status $[n(\%)]$} & Single & $28(45.9)$ & $31(50.8)$ & \multirow[t]{3}{*}{$\chi^{2}(2)=1.10, p=.58$} \\
\hline & Married & $30(49.2)$ & $25(41.0)$ & \\
\hline & Separated/divorced & $3(4.9)$ & $5(8.2)$ & \\
\hline \multirow[t]{5}{*}{ Religion $[n(\%)]$} & None & $40(65.6)$ & $42(68.9)$ & \multirow[t]{5}{*}{$\chi^{2}(4)=1.42, p=.84$} \\
\hline & Buddhism & $15(24.6)$ & $14(23.0)$ & \\
\hline & Catholicism & $3(4.9)$ & $1(1.6)$ & \\
\hline & Christianity & $2(3.3)$ & $2(3.3)$ & \\
\hline & Other & $1(1.6)$ & $2(3.3)$ & \\
\hline
\end{tabular}

baseline. By using two one-sided tests for equivalence (TOST; Rogers et al. 1993), the various psychological scores and age between these two groups were found to be equivalent at baseline $(p<.05$, the equivalence criterion was set at $20 \%$ of the ATP group's mean).

\section{Procedure}

The trial was conducted and reported according to the guidelines of the Consolidated Standards of Reporting Trials (CONSORT) 2010 (Moher et al. 2012; Schulz et al. 2010). The selection criteria were not disclosed to the participants to avoid the concealment of information. The screening was based on the information that the participants provided in the online registration form. If there was any missing or insufficient information that related to their eligibility, telephone or face-to-face interviews were arranged to acquire further information. All the participants who met the inclusion criteria were listed with an assigned number on an excel sheet. An independent and blind researcher, who was not part of this research project team, was provided with the assigned number only to perform the random selection by generating random numbers in a computer. Next, all the eligible participants were invited to attend a briefing session after the random selection. In both the briefing session and the recruitment process, the participants were informed that the ATP is a Buddhist teaching-based intervention that comprises Buddhist teachings and practice. During the briefing session, the research procedures and requirements for attending the ATP were introduced. The participants were told that they must commit to engaging in a daily meditation practice and attending the entire ATP. The participants then completed an informed consent form and questionnaire if they agreed to participate in the research. All answers were checked before the participants left the meeting to ensure that there were no missing data. After the completion of the baseline assessment, the group allocation was further processed by giving the participants' assigned numbers to the same independent researcher who performed the random selection. Because female participants were overrepresented, the participants were stratified by gender and assigned to either the intervention group or the control group by generating random numbers in the computer. The participants were blinded to their allocation condition until after the completion of the baseline assessment.

\section{Intervention}

As explained above, the theoretical foundation of the ATP is based on the teachings in the Mahayana Buddhist text, the Sandhinirmochana Sūtra. In the Sūtra, the Buddha declared that if a practitioner wishes to begin practicing meditation in the Mahayana tradition, his or her practice should be based on the texts of Mahayana teachings and depends on the commitment to attaining perfect enlightenment (bodhicitta) (Keenan 2000, p. 51). The texts of the Mahayana teachings serve as the foundation and guidelines for developing the wisdom of nonattachment. The commitment to attaining perfect enlightenment (bodhicitta) arises from the compassion to save all beings from suffering. Therefore, the primary objective of the ATP is to enhance the participants' wisdom of nonattachment and compassion. We posit that by enhancing these two qualities of the wisdom of nonattachment and compassion, the participants' stress management and psychological wellbeing will improve.

In the ATP, the development of both the wisdom of nonattachment and compassion follows the three pedagogical steps 
of developing wisdom, as taught in the Sandhinirmochana Sütra. Under this pedagogical system, practitioners must first learn and memorize core Buddhist teachings to develop the wisdom of nonattachment that is realized by learning; next, they must repeatedly focus on and contemplate the meaning of these teachings to develop the wisdom of nonattachment that is realized by thinking. Finally, they must accordingly understand these teachings and practice to develop the wisdom of nonattachment that can be realized by practice (Keenan 2000, p. 63; Sik and Wu 2015).

The core Buddhist teaching that the participants learned and memorized in the ATP was the mantra "Om mani padme hüm," and the meaning of the mantra is summarized as follows:

The pearl of compassion is entirely auspicious,

The wisdom lotus cannot be tainted,

All phenomena arise interdependently and are therefore intrinsically nonarising,

May all beings be able to realize these truths.

To develop compassion, the participants were first guided to see the universality of suffering for themselves and other people; then, they were guided to send compassionate wellwishes to themselves, other people and all sentient beings in succession. For the development of the wisdom of nonattachment and to overcome the "ontological addiction" or the attachment to the concept of "self" or "I," which are the sources of human suffering, the participants were guided to systematically study and recognize the emptiness or essencelessness characteristics of the three types of characteristics of phenomena to develop the wisdom of nonattachment. Lecture notes, revision exercises and corrections to the revision exercises were also incorporated into the program to help the participants to think and reflect on what they had learned in the workshops to develop the wisdom of nonattachment that can be realized by thinking. Finally, the participants gained insight into how concepts and attachment arise and cease through experiential activities, and they were taught to develop compassion and the wisdom of nonattachment through the meditative practice of tranquility (śamatha) and observation (vipaśyana) to develop the wisdom of nonattachment that can be realized through practice.

The ATP consists of six 3-h workshops plus two whole-day weekend retreats ( $7 \mathrm{~h}$, excluding the lunch hour; see Table 2 ). Each workshop began (except for the first session) with sessions of approximately $20 \mathrm{~min}$ of sharing to provide opportunities for the participants to share or ask any questions that related to their meditation experiences in the homework assignment. At the end of each workshop (except for the last session), revision exercises were done, corrections to the revision exercises were made and the weekly meditation homework assignment was given out. In addition, lectures, meditation practices, experiential and reflective learning activities and group discussions were employed to enrich the learning experience. Each participant received audio tracks with guided meditations, handouts, and homework assignment record worksheets to support their learning.

To maintain the consistency of the teaching of the program, HHS, the prime developer of the ATP, conducted all the workshops. HHS has more than 30 years of meditation experience. $\mathrm{He}$ is also the 45th generation lineage holder of Ling-ji (臨濟 宗) and the 10th generation lineage holder of the Gui-yang (溈 仰宗) Schools of Ch'an.

\section{Measures}

The 10-item Perceived Stress Scale (PSS-10; Cohen et al. 1983) was used to assess the participants' level of psychological stress. This scale measures the degree to which the situations in one's life are appraised as stressful (Cohen and Williamson 1988). The PSS-10 has 10 items and uses a 5point Likert scale that ranges from 0 (never) to 4 (very often). Lower scores indicate lower stress levels. In this sample, the Cronbach's alpha coefficient was .88 at baseline, .87 at posttest, and .89 at follow-up.

The 13-item Sense of Coherence Scale (SOC-13), which is also known as the Orientation to Life Questionnaire (OLQ13), was used to measure the ability to cope with stress (SOC13; Antonovsky 1987). According to Antonovsky (1987), a person with a strong sense of coherence can understand and manage life's difficulties and stress to stay well; such a person will perceive the world as coherent, predictable, comprehensible, and meaningful. Therefore, in this study, a sense of coherence is used to assess one's ability to manage stress. The SOC-13 consists of 13 items and uses a 7-point Likert scale that ranges from 1 to 7 . Higher scores indicate a better ability to handle stress. The Cronbach's alpha coefficient was .87 at baseline, .90 at posttest, and .89 at follow-up in this study.

The 12-item General Health Questionnaire was used to assess the participants' psychological well-being (GHQ-12; Goldberg 1992). The GHQ-12 is widely used as a measure of general psychological health. It has 12 items and uses a 4point Likert scale that ranges from 0 to 3 . Lower scores indicate better psychological well-being. In the present study, the internal consistency was very good, as shown by the Cronbach's alpha at .89 at baseline, .91 at posttest, and .90 at follow-up.

The Nonattachment Scale was used to measure the participants' level of wisdom in the form of nonattachment (NAS; Sahdra et al. 2010). This is a psychological measure of the Buddhist state or trait of nonattachment. The construct of nonattachment is characterized by having a subject quality of a relative absence of fixation on ideas, images, or sensory objects, and not feeling an 
Table 2 Outline of Awareness Training Program (ATP)

\begin{tabular}{|c|c|c|}
\hline Session & Key teachings & Themes and contents \\
\hline 1. & Bodhicitta & Generating aspiration and commitment \\
\hline 2. & $\begin{array}{l}\text { Awareness, mindfulness, and } \\
\text { Buddha nature }\end{array}$ & Learning and experiencing the power of awareness \\
\hline 3. & Compassion & Recognizing and understanding the universality of suffering to generate compassion \\
\hline \multirow[t]{2}{*}{4.} & Compassion & Whole-day retreat (1): cultivating compassion for oneself and others \\
\hline & Wisdom & Whole-day retreat (2): cultivating the wisdom of nonattachment through observation of the concept of self \\
\hline 5. & Wisdom of nonattachment & $\begin{array}{l}\text { Developing the wisdom of nonattachment through understanding and experiencing the three types of } \\
\text { characteristics (三自性) and the three types of essencelessness characteristics (三種無自性性) of phenomena }\end{array}$ \\
\hline 6. & Bodhicitta & Discovering the meaning of life \\
\hline 7. & Solidify learning experiences & Summarizing the learning experience and developing the sustainability of practice \\
\hline
\end{tabular}

internal pressure to get, hold, avoid, or change circumstances or experiences (Sahdra et al. 2010). It has 30 items and uses a 6-point Likert scale that ranges from 1 to 6 . Higher scores indicate a higher level of nonattachment. The scale was translated into Chinese by using the back-translation method, and it has an excellent internal consistency, as shown by a Cronbach's alpha at .94 at baseline, .95 at posttest, and .96 at follow-up in this study.

A pop quiz was used at the 3-month follow-up to assess the knowledge proficiency of the Buddhist teachings and meditation methods that the participants had learned in the program. The participants of the intervention group were asked to answer 20 multiple-choice questions, and the participants who obtained ten or more correct answers were considered to have passed. The following are some of our sample questions: "What is the meaning of 'Om'?" and "What are the obstacles that you encountered while meditating?" Moreover, the participants were also asked to indicate whether they have continued to practice meditation; if yes, they were asked about the frequency of their meditation practice and whether they had reviewed the content or handouts of the ATP within the 3 months after the intervention.

\section{Data Analyses}

The differences in the demographic characteristics across the groups at baseline were compared by conducting chi-squared tests for the categorical variables and independent sample $t$ tests for the continuous variables with the Statistical Package for Social Sciences (SPSS Inc., Version 23.0, Chicago, USA) software. In addition, the equivalence of the psychological scores and age between groups at baseline were tested by conducting TOST using XLSTAT version 2018.3 ("testing equivalence with TOST in excel tutorial," 2017).

Statistical analyses of the outcome measures were performed with SPSS version 23.0. Both an intention-to-treat (ITT) analysis and per-protocol (PP) analysis were performed to assess the robustness of the results. ITT was the primary analysis, and PP was the supporting analysis. For the ITT analysis, a conservative method was used that included all the participants once they were randomized, regardless of whether they had received the intervention (Armijo-Olivo et al. 2009, p. 40). A Little's Missing Completely At Random (MCAR) test was performed to assess whether the data were missing completely at random. The dropout data of the outcome variables at posttest and 3-month follow-up were addressed by multiple imputation. The total score for each individual participant's scale was calculated after each missing variable of the outcome measures was imputed. The pooled results of the $F$ values, $p$ values, and degrees of freedom of ANOVAs and analyses of covariance (ANCOVAs) were obtained by following the guidelines and procedures suggested by Van Ginkel (2014). For the PP analysis, listwise deletion was adopted to address the dropout data.

Both the ITT analysis and the PP analysis involved the following statistical analyses. A mixed betweenwithin subjects ANOVA was conducted to assess the effectiveness of the ATP on the participants' scores on each level of PSS, SOC, GHQ, and NAS across three between-group time points. Afterwards, the intervention effect (a comparison of the differences between the pretest and posttest scores) and maintenance effect (a comparison of the differences between the pretest and post-3month follow-up scores) were examined. The intervention effect and maintenance effect were examined by performing a two-way mixed ANOVA to compare the interaction effect between groups and among times on each of the outcome measures. For any significant interaction that was found, an ANCOVA was conducted to examine the group effect after adjusting the baseline. The pooled effect size of the imputed data set of the ANOVAs and ANCOVAs were manually calculated by following the rules suggested by Harel (2009). All effects were deemed to be significant when the $p$ value fell below the .05 significance level (two-tailed). 


\section{Results}

\section{Attrition and Attendance}

The number of dropouts in this study was $12(10 \%)$ of which 7 and 5 were from the intervention group and the control group, respectively. Three participants from the intervention group and 2 participants from the control group immediately dropped out after receiving the notification of their allocated ATP schedule because they were not available during the allocated timeslot. Other reasons for dropping out include a loss of interest in participating in the research, a busy work schedule, illness, a loss of contact, and a conflict between work and program schedules, although the participant wanted to continue to participate (see Fig. 1). Of the participants who attended the ATP, more than $90 \%(91.2 \%)$ attended at least seven out of eight sessions (of which six were 3 -h workshops and two were all-day retreats).

\section{Analysis of the Self-Reported Outcome Measures}

Regarding the dropout data, a nonsignificant Little's MCAR test, $\chi^{2}=302.424(\mathrm{df}=392, p=1)$, indicated that the data were missing completely at random (Little 1988). Twenty imputed data sets were produced for data analysis and for pooling the results of the ITT analysis. The results of the ITT $(n=122)$ and PP analyses $(n=110)$ are summarized in Table 3. For the results of the ITT analysis, the improvements of the average levels of PSS (pre: ATP: $M=22.15$; control: $M=20.75$; post: ATP: $M=18.15$; control: $M=19.83$; post-3: ATP: $M=17.58$; control: $M=19.77$ ), SOC (pre: ATP: $M=$ 52.89; control: $M=54.00$; post: ATP: $M=57.64$; control: $M=54.67$; post-3: ATP: $M=60.35$; control: $M=55.34$ ), GHQ (pre: ATP: $M=15.98$; control: $M=15.61$; post: ATP: $M=12.01$; control: $M=15.61$; post-3: ATP: $M=11.59$; control: $M=14.75$ ), and NAS (pre: ATP: $M=111.49$; control: $M=115.46$; post: ATP: $M=127.87$; control: $M=115.58$; post-3: ATP: $M=128.77$; control: $M=118.36$ ) of the ATP group were greater than these levels for the controls from the pretest to the posttest and from the pretest to the post-3month follow-up. The two-way mixed ANOVA analysis indicated a highly significant group (intervention, control) by time (pretest, posttest, post-3-month) interaction for the overall interaction effects in all outcome variables (PSS: $F(2,231.89)=$ 14.96, $p<.001, \eta p 2=.12$; SOC: $F(2,237.10)=7.48$, $p<.001, \eta p 2=.06$; GHQ: $F(2,235.94)=15.10, p<.001$, $\eta p 2=.12$; NAS: $F(2,237.38)=17.29, p<.001, \eta p 2=.13)$. The results showed that there were statistically significant improvements across the three between-group time points in all outcome variables for the ATP group over the controls, with an overall medium effect size in SOC, a medium to large effect size in PSS, GHQ, and NAS.

\section{Treatment and Maintenance Effects}

For the treatment effect, the results of the two-way mixed ANOVA indicated a significant interaction between group (intervention, control) and time (pretest, posttest) in all outcome variables $(\mathrm{PSS}: F(1,113.79)=19.78, p<.001, \eta p$ $2=.15 ;$ SOC: $F(1,115.60)=5.78, p=.018, \eta p 2=.05$; GHQ: $F(1,112.89)=22.87, p<.001, \eta p 2=.16$; NAS: $F(1$, $117.40)=24.41, p<.001, \eta p 2=.17)$. Further analysis was conducted by using a one-way ANCOVA to determine the statistically significant difference between the ATP and control groups on the posttest scores after controlling for the pretest score to investigate the between-group (group) effect. The results showed significant differences in the ATP group in all outcome variables (PSS: $F(1,111.16)=18.10, p<.001$, $\eta p$ $2=.14$; SOC: $F(1,114.06)=5.69, p=.019, \eta p 2=.05$; GHQ: $F(1,110.83)=24.81, p<.001, \eta p 2=.18$; NAS: $F(1$, $116.11)=27.34, p<.001, \eta p 2=.19)$. The results showed statistically significant improvements over the controls across the two between-group time points and between-group from the pretest to the posttest in the ATP group for all outcome measures, with a small to medium effect size in SOC and a large effect size in PSS, GHQ, and NAS.

For the maintenance effect, the results of the two-way mixed ANOVA indicated a highly significant interaction between group (intervention, control) and time (pretest, post-3month) in all outcome variables (PSS: $F(1,111.14)=22.06$, $p<.001, \eta p 2=.16 ;$ SOC: $F(1,116.50)=12.93, p<.001, \eta p$ $2=.10$; GHQ: $F(1,115.33)=18.31, p<.001, \eta p 2=.13$; NAS: $F(1,116.17)=18.62, p<.001, \eta p 2=.14)$. Further analysis was conducted to investigate the between-group (group) effect by using a one-way ANCOVA to determine the statistically significant difference between the ATP and control groups in the post-3-month scores after controlling for the pretest score. The results showed highly significant differences in the ATP group in all outcome variables (PSS: $F(1,108.86)=19.73, p<.001, \eta p 2=.15$; SOC: $F(1$, $114.96)=14.93, p<.001$, ๆp $2=.11$; GHQ: $F(1,113.49)=$ $21.25, p<.001, \eta p 2=.15$; NAS: $F(1,114.73)=19.12, p$ $<.001, \eta p 2=.14)$. The results showed statistically significant improvements over the controls across the two between-group time points and between-group from the pretest to the 3month follow-up in the ATP group for all outcome measures. The effect size of the interaction effect was medium to large in SOC and GHQ and large in PSS and NAS, whereas the between-group effect size was medium to large in SOC and large in PSS, GHQ, and NAS. The results of the PP analysis $(n=110)$ were similar to the results of the ITT analysis presented above but with higher significant differences and larger effect sizes (see Table 3).

Nearly all of the participants (98.1\%) from the intervention group passed the pop quiz at the post-3-month follow-up. Forty-one $(75.9 \%)$ of the 54 participants have continued to 
Excluded ( $\mathrm{n}=298)$

- Did not meet inclusion criteria $(n=254)$

- Unavailability to participate the whole or part of $\operatorname{ATP}(n=11)$

- Unavailable to come to the briefing session and baseline assessment $(\mathrm{n}=4)$

- Lost contact $(n=2)$

- Declined to participate in the research $(n=1)$

- Did not include because the optimal group size and target sample size were met $(n=26)$

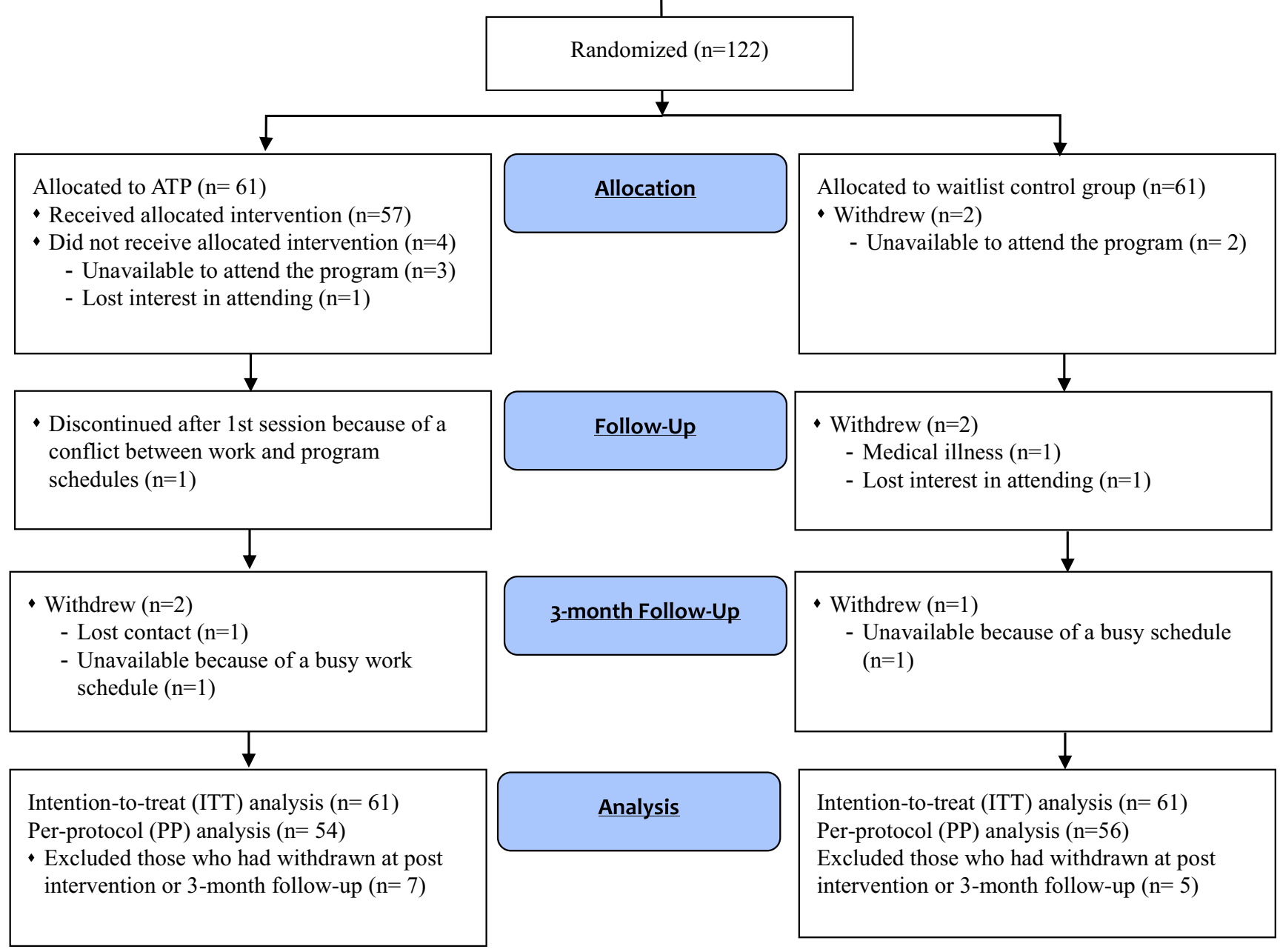

Fig. 1 The Consolidated Standards of Reporting Trials (CONSORT) diagram that shows the flow of participants 


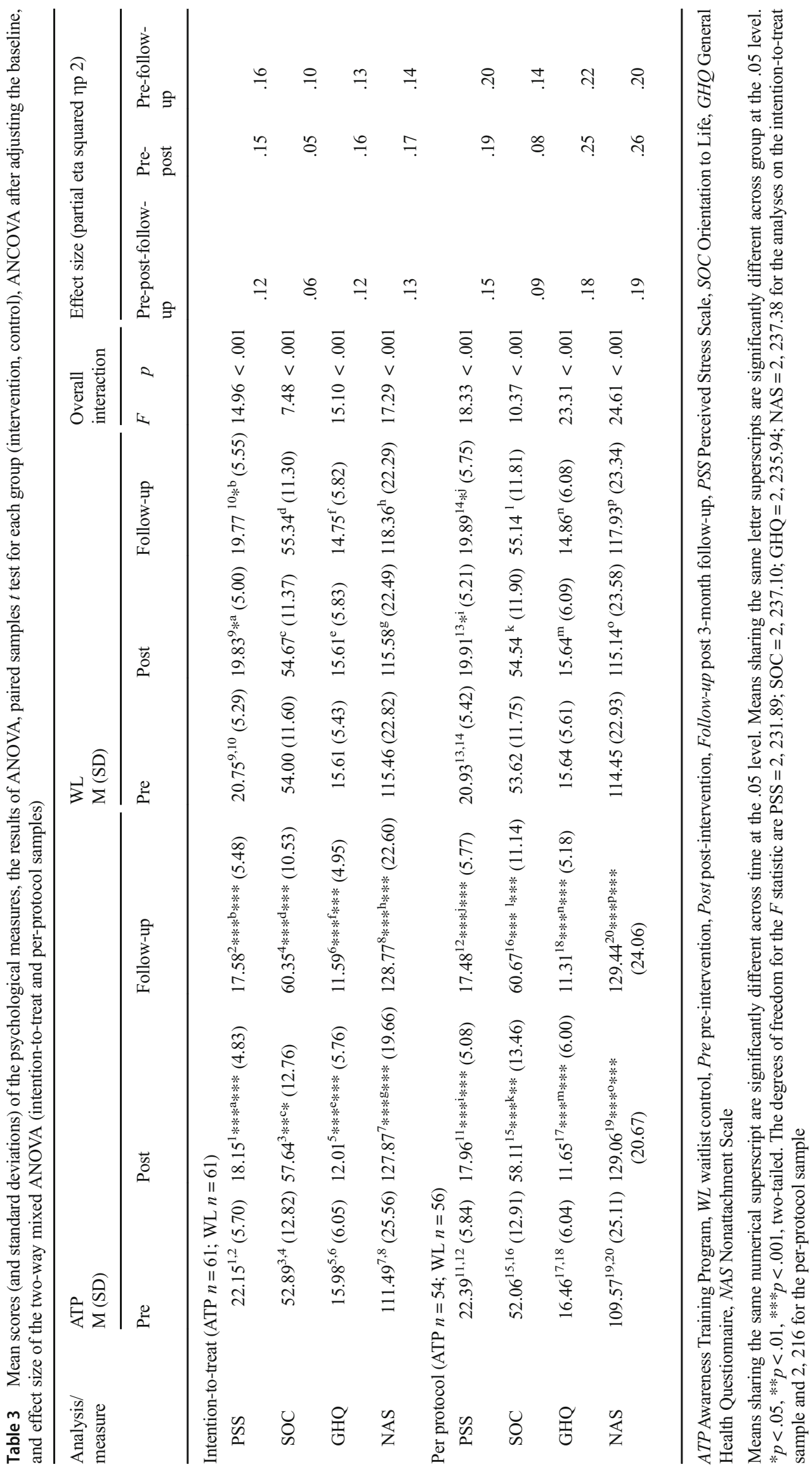


practice meditation, and $27(50 \%)$ have reviewed the content or handouts of the ATP within the post-follow-up period. More than one fifth $(20.8 \%)$ of them meditated every day, and more than two thirds $(63 \%)$ of them maintained the practice of weekly meditation within the post-follow-up period.

\section{Mediation Analysis}

We hypothesized that wisdom in the form of nonattachment would be the mediating factor of the outcome measures. Specifically, the intervention group's level of NAS mediated the effect of the ATP on PSS, SOC, and GHQ. Therefore, we examined the mediator of the ATP, i.e., the changes in NAS, for each outcome variable at posttest and at 3-month followup after controlling for their respective baseline levels by using the four-step approach proposed by Baron and Kenny (1986). The four steps that are essential to establish mediation are as follows: the first step is to illustrate that the causal variable (i.e., the intervention) is correlated with the outcome variable (i.e., the post-PSS, post-SOC, post-GHQ, post-3 PSS, post-3 SOC, and post-GHQ scores); the second step is to show that the causal variable is correlated with the mediator (in this study, i.e., the changes in the pre-post NAS scores and the changes in the pre-post-3 NAS scores); the third step is to demonstrate that the mediator affects the outcome variable while controlling for the causal variable; and the fourth step is to establish that the mediator mediates the relationship between the causal variable and the outcome variable, and the effect of the causal variable on the outcome variable that controls for the mediator should become nonsignificant or less significant (Baron and Kenny 1986; Kenny 2016). A series of hierarchical linear regression analyses were conducted. In addition, the bootstrapping method (Hayes 2018a) was also performed as an alternate method to support the results. Bootstrapping was run with the PROCESS macro v3.0 by Andrew F. Hayes (2018b) in SPSS Inc., Version 25.0, Chicago, USA. The bootstrap was based on 5000 bootstrap samples with a bias-corrected accelerated method to estimate the $95 \%$ confidence interval (CI). If zero is not included within the estimated CI, it indicates a significant mediation effect at the 0.05 level.

\section{The Mediating Effect of Nonattachment at Posttest and at Post-3-Month Follow-Up}

The following is a description of the hierarchical linear regression analyses of the mediating effect of nonattachment at posttest. First, the participants of the ATP group were observed to have significant changes in the outcome variable at posttest, after accounting for the baseline levels of the outcome variable (post-PSS: $B=-2.99, p<.001$; post-SOC: $B=4.59, p=.004$; post-GHQ: $B=-4.52, p<.001)$. Second, the analyses indicated that the participants of the ATP group increased their levels of nonattachment from baseline to posttest $(B=18.16$, $p<.001)$. Third, the changes in the levels of nonattachment from baseline to posttest were found to be a significant predictor of the post-outcome variable after accounting for the baseline levels of the outcome variable and intervention (post-PSS: $B=-0.092, p<.001$; post-SOC: $B=0.14$, $p=.007$; post-GHQ: $B=-0.094, p<.001)$. Finally, we regressed the post-outcome variable on the baseline levels of the outcome variable in the first block and experimental condition, and the pre-post changes in the levels of nonattachment were included as predictors in the second block. For the PSS outcome variable, we observed that the previously significant effect of the ATP on lower levels of PSS at posttest became less significant $(B=-1.49, p=.018)$, while the pre-post changes in the levels of nonattachment continued to be a significant predictor $(B=-0.090, p<.001)$. For the SOC outcome variable, we observed that the previously significant effect of the ATP on increasing the levels of SOC at posttest became nonsignificant $(B=2.11, p=.24)$, while the pre-post changes in the levels of nonattachment continued to be a significant predictor $(B=0.14, p=.006)$. For the GHQ outcome variable, we observed that the previously significant effect of the ATP on lower levels of GHQ at posttest became less significant $(B=-2.87, p=.001)$, while the pre-post changes in the levels of nonattachment continued to be a significant predictor $(B=-0.095, p<.001)$. The results of the hierarchical linear regression analyses showed that the conditions that are required to establish mediation between the mediator and the respective outcome measure were met (Fig. 2). For the mediating effect of nonattachment at the post-3 follow-up, the results of the hierarchical linear regression analyses were similar to the results at posttest, which also demonstrated that the conditions that are required to establish mediation between the mediator and the respective outcome measure were met (Fig. 2). Therefore, NAS was a mediator of the treatment effects and maintenance of the treatment effects across all outcome measures (PSS, SOC, and GHQ) at posttest and 3month follow-up.

Bootstrapping, which is another popular method for mediation analysis, was also conducted. The bootstrap results showed that NAS significantly mediated the relationship between the ATP and all outcome measures (see Fig. 2), which supports the findings of the hierarchical linear regression analyses. In addition, the results of Sobel's test were also consistent with the results of the above analyses.

\section{Discussion}

We assessed the effectiveness of the ATP, a semisecularized group-based intervention based on the Mahayana Buddhist teaching that is "textually aligned" with "coherent theory and praxis." By employing lectures, experiential and reflective 
Fig. 2 Mediation diagrams that summarize the hierarchical linear regression and bootstrap analyses by using nonattachment as a mediator of the effect of the intervention on stress, sense of coherence, and psychological well-being. ATP Awareness Training Program, PSS Perceived Stress Scale, SOC Orientation to Life, GHQ General Health Questionnaire, NAS

Nonattachment Scale, $B S$ results of bootstrap analyses, $H L R$ results of hierarchical linear regression analyses; $* p<.05, * * p<.01$, $* * * p<.001$. If zero is not included within the estimated confidence interval, it indicates a significant mediation effect at the 0.05 level. Numbers in parentheses are the $B$ after controlling for the mediator. Numbers in square brackets are the lower level confidence interval and upper level confidence interval

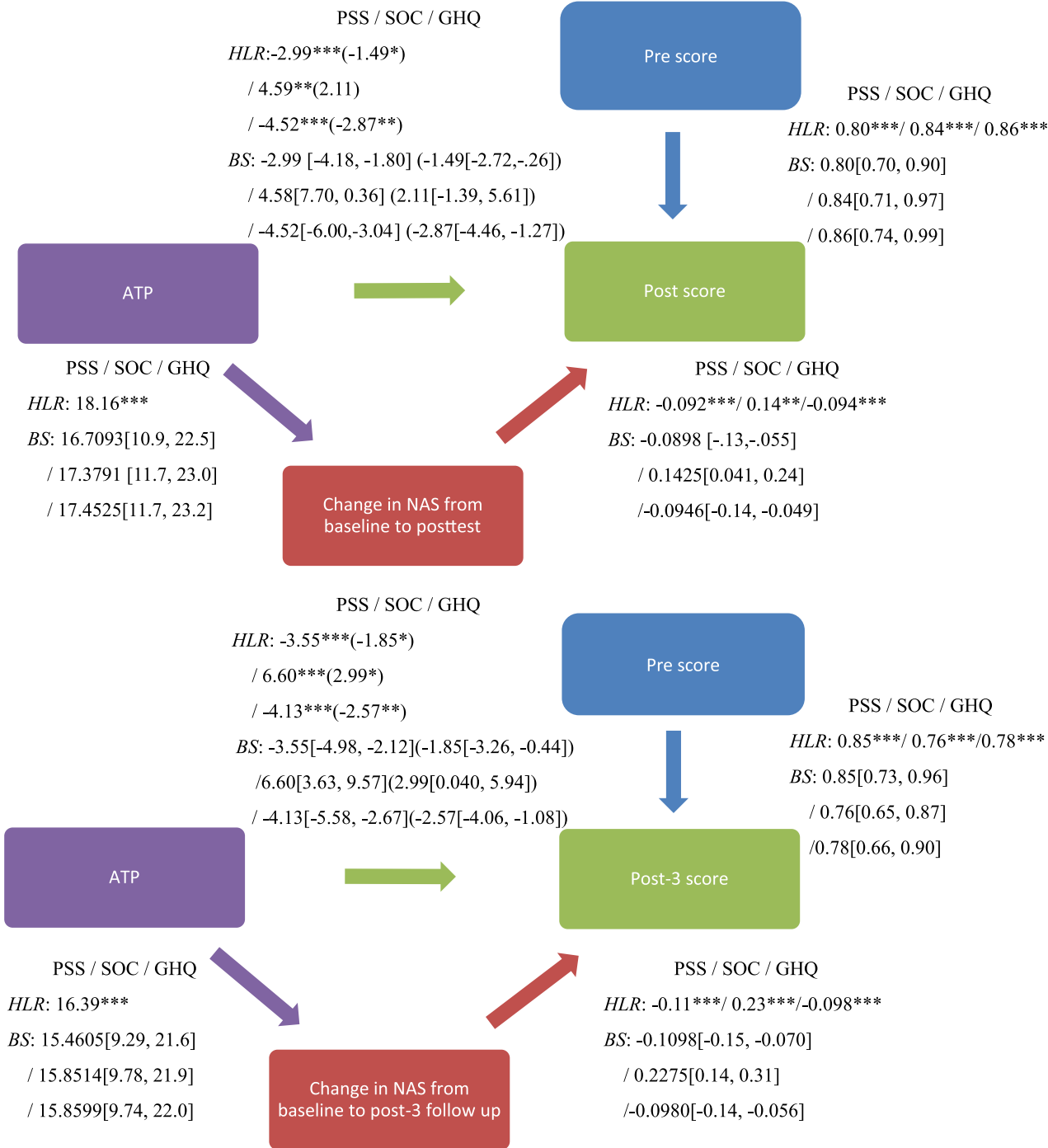

learning activities, meditation homework assignments, group discussions, and revision exercises, the ATP has been demonstrated to be effective in improving stress levels, a sense of coherence, psychological well-being, and the wisdom of nonattachment for middle-aged working adults, and the therapeutic improvements were maintained at 3-month follow-up. The outcomes were aligned with the hypothesis, and the experimental group demonstrated significant improvements over the control group on all the self-reported outcome measures.

Our findings are generally consistent with the previous studies of various forms of MBIs that show significant improvement in the levels of stress reduction, stress management and psychological well-being in working adults (Foureur et al. 2013; Shonin et al. 2014; Taylor et al. 2016; Wallmark et al. 2013) and middle-aged adults (Lee and Bang 2010). In general, the effect sizes of the interaction effect between group and time on each of the outcome measures were from medium to strong. The between-group effect size and within-group effect size in this study are also generally consistent with the findings of a recent meta-analysis of MBI studies in reducing the psychological distress of working adults (Virgili 2015).

The data from our study indicated that wisdom in the form of nonattachment (NAS) served as the key component of ATP to mediate the improvement of the levels of perceived stress (PSS), sense of coherence (SOC), and psychological wellbeing (GHQ). This supported our hypothesis that wisdom in the form of nonattachment is an important and effective mediator to reduce an individual's delusions and suffering. These findings endorse Sahdra's assertion (Sahdra et al. 2010, p. 125 ) that "release from mental fixation (nonattachment) is thought to encourage more objective perception, greater compassion, reduced selfishness, and release from, or letting go of, what Buddhist call 'afflictive' emotion, thus alleviating suffering."

A significant maintenance effect was also found at 3-month follow-up. We attribute this significant maintenance effect of the ATP to the effectiveness of the three pedagogical steps of developing the wisdom of nonattachment as taught in the 
Sandhinirmochana Sütra. Under this pedagogical system, practitioners must first learn and memorize the teaching, then repeatedly focus and contemplate on its meaning and finally accept and practice accordingly. It would seem that the cognitive learning and reflective learning helped the participants to retain their knowledge acquired from the program, as demonstrated in the high pass rate of the pop quiz at 3-month follow-up. Moreover, many participants maintained their meditation practice within the post-follow-up period. The findings of this study, together with the school-based contemplative education program (Sik and Wu 2015), support that the Buddhist pedagogy of the three wisdoms may be a desirable method to foster participants' learning experience.

Another factor that might have contributed to the significant maintaining effect of the ATP is the importance of NAS to psychological well-being. Our findings reveal that changes in NAS mediate the maintenance impact of the ATP on participants' stress levels, sense of coherence, and psychological well-being. Our findings are consistent with the only other group-based study for adults that used NAS as an outcome measure in an interventional study (Van Gordon et al. 2017). The findings of Van Gordon et al.'s study also demonstrated that nonattachment was enhanced both at post and at post-follow-up, and it continued to positively mediate the psychological distress outcome for participants with fibromyalgia.

In addition, the ATP and the findings of this study may inform second-generation MBIs' theory and research. In this study, we posited that the developing trend of the "second-generation MBIs," that is, to formulate an MBI by referring back to its Buddhist origins, could be further developed by formulating an intervention that systematically combines a Buddhist theoretical model with a compatible meditation practice. The development of the ATP and the significant findings of this study demonstrate that a semisecular group intervention based on Mahayana Buddhist teaching that adopts a textually aligned approach with a coherent theory and praxis could be an effective means to enhance the psychological health and well-being of people in contemporary society. Since this is the first attempt to develop and investigate an intervention that adopts a textually aligned approach, these positive findings may inform researchers' future approaches to formulate and design Buddhist-derived interventions.

\section{Limitations}

This study has several limitations. First, the use of middleaged working adults as the sample may limit the generalizability of the findings. Future studies should implement this program for different populations. Second, the therapeutic gains may also be partially attributed to some nonspecific factors that cannot be excluded, for example, therapeutic alliance and instructor competence. Moreover, the improved outcome may be attributed to simple expectancy effects because a waitlist control instead of an active control is used as the comparison group. Furthermore, by using a waitlist control group and not making a direct comparison with other interventions, the "extra benefit," if any, of incorporating "Mahayana Buddhist teachingbased," "textually aligned," and "coherent theory and praxis" into an intervention could not be verified. An active control group could be employed in future research. In addition, the maintenance effect was assessed at 3-month follow-up in this study. Further investigation of the effectiveness of the ATP could go beyond the 3 months after the intervention. In this study, the ATP has only been applied to people in the East; the suitability of using the ATP in the West still needs to be further explored. Although it adopted a semisecular approach and simultaneously informed participants of its Buddhist root and Buddhist content, the ATP may only be suitable for people who are comfortable with its Buddhist background. Therefore, in a highly nonBuddhist religious society, the ATP may not be easily accepted.

Authors' Contributions BWYW designed, conceived, and executed the study; developed the program content of the intervention; collected, analyzed, and interpreted the data; and collaborated in the writing of the paper and editing the final manuscript. JG randomly checked the accuracy of the self-reported data and collaborated in editing the final manuscript. HL collaborated in editing the final manuscript and co-analysis on the equivalence testing. HHS designed and conceived the study, developed the theoretical model and the program content of the intervention, was the ATP instructor, and collaborated in the writing of the paper and editing the final manuscript.

Funding This research was funded by the Li Ka Shing Foundation.

\section{Compliance with Ethical Standards}

This study had received ethical approval from the Human Research Ethics Committee for Non-Clinical Faculties at The University of Hong Kong. Informed consent was obtained from all individual participants who were included in the study. Moreover, an anonymous data set of the participants' name, date of birth, email, and contact number was used for all data analysis and has since been deleted.

Conflict of Interest The authors declare that they have no conflict of interest.

Open Access This article is distributed under the terms of the Creative Commons Attribution 4.0 International License (http:// creativecommons.org/licenses/by/4.0/), which permits unrestricted use, distribution, and reproduction in any medium, provided you give appropriate credit to the original author(s) and the source, provide a link to the Creative Commons license, and indicate if changes were made.

Publisher's Note Springer Nature remains neutral with regard to jurisdictional claims in published maps and institutional affiliations. 


\section{References}

Antonovsky, A. (1987). The Jossey-Bass social and behavioral science series and the Jossey-Bass health series. Unraveling the mystery of health: How people manage stress and stay well. San Francisco: Jossey-Bass.

Armijo-Olivo, S., Warren, S., \& Magee, D. (2009). Intention to treat analysis, compliance, drop-outs and how to deal with missing data in clinical research: a review. Physical Therapy Reviews, 14(1), 3649.

Baron, R. M., \& Kenny, D. A. (1986). The moderator mediator variable distinction in social psychological-research - conceptual, strategic, and statistical considerations. Journal of Personality and Social Psychology, 51(6), 1173-1182.

Bodhi, B. (2011a). Dhamma and non-duality. Retrieved from http://www. accesstoinsight.org/lib/authors/bodhi/bps-essay_27.html. Accessed 27 Aug 2016.

Bodhi, B. (2011b). What does mindfulness really mean? A canonical perspective. Contemporary Buddhism, 12(01), 19-39.

Chiesa, A., \& Malinowski, P. (2011). Mindfulness-based approaches: are they all the same? Journal of Clinical Psychology, 67(4), 404-424.

Cleary, T. (1995). Buddhist yoga: a comprehensive course. Boston: Shambhala.

Cohen, S., \& Williamson, G. (1988). Perceived stress in a probability sample of the United States. In S. Spacapan \& S. Oskamp (Eds.), The social psychology of health (pp. 31-67). Newbury Park, CA: Sage.

Cohen, S., Kamarck, T., \& Mermelstein, R. (1983). A global measure of perceived stress. Journal of Health and Social Behavior, 385-396.

Foureur, M., Besley, K., Burton, G., Yu, N., \& Crisp, J. (2013). Enhancing the resilience of nurses and midwives: pilot of a mindfulness-based program for increased health, sense of coherence and decreased depression, anxiety and stress. Contemporary Nurse, 45(1), 114-125.

Franz, F., Erdfelder, E., Lang, A. G., \& Buchner, A. (2007). G*power 3: a flexible statistical power analysis program for the social, behavioral, and biomedical sciences. Behavior Research Methods, 39(2), 175191.

Franz, F., Erdfelder, E., Lang, A. G., \& Buchner, A. (2009). Statistical power analyses using $\mathrm{g}^{*}$ power 3.1: tests for correlation and regression analyses. Behavior Research Methods, 41, 1149-1160.

Goldberg, D. (1992). General health questionnaire (GHQ-12). Windsor: Nfer-Nelson

Harel, O. (2009). The estimation of $\mathrm{R}^{2}$ and adjusted $\mathrm{R}^{2}$ in incomplete data sets using multiple imputation. Journal of Applied Statistics, 36(10), $1109-1118$

Hayes, A. F. (2018a). Introduction to mediation, moderation, and conditional process analysis second edition: a regression-based approach. New York: Guilford Press.

Hayes, A. F. (2018b). The process macro for SPSS and SAS. Retrieved from http://processmacro.org/download.html. Accessed 25 July 2018.

Kabat-Zinn, J. (2015). Mindfulness has huge health potential-but McMindfulness is no panacea. The Guardian. Retrieved from https://www.theguardian.com/commentisfree/2015/oct/20/ mindfulness-mental-health-potential-benefits-uk. Accessed 28 March 2016.

Keenan, J. P. (2000). The scripture on the explication of underlying meaning. California: Numata Center for Buddhist Translation and Research.

Kenny, D. A. (2016). Mediation. Retrieved from http://davidakenny.net/ $\mathrm{cm} /$ mediate.htm. Accessed 1 March 2016.

Kochumuttom, T. A. (1999). A Buddhist doctrine of experience. A new translation and interpretation of the works of Vasubandhu the Yogacarin. Delhi: Motilal Banarsidass.
Lee, W. K., \& Bang, H. J. (2010). The effects of mindfulness-based group intervention on the mental health of middle-aged Korean women in community. Stress and Health, 26(4), 341-348.

Little, R. J. (1988). A test of missing completely at random for multivariate data with missing values. Journal of the American Statistical Association, 83(404), 1198-1202.

Moher, D., Hopewell, S., Schulz, K. F., Montori, V., Gøtzsche, P. C., Devereaux, P. J., et al. (2012). CONSORT 2010 explanation and elaboration: updated guidelines for reporting parallel group randomised trials. International Journal of Surgery, 10(1), 28-55.

Monteiro, L. M., Musten, F., \& Leth-Steensen, C. (2018). Effect of mindfulness on value incongruence: a pilot study. Mindfulness. Advance of Print.

Rogers, J. L., Howard, K. I., \& Vessey, J. T. (1993). Using significance tests to evaluate equivalence between 2 experimental groups. Psychological Bulletin, 113(3), 553-565.

Sahdra, B. K., Shaver, P. R., \& Brown, K. W. (2010). A scale to measure nonattachment: a Buddhist complement to Western research on attachment and adaptive functioning. Journal of Personality Assessment, 92(2), 116-127.

Schulz, K. F., Altman, D. G., \& Moher, D. (2010). CONSORT 2010 statement: updated guidelines for reporting parallel group randomised trials. BMC Medicine, 8(1), 18.

Shonin, E., \& Van Gordon, W. (2015). The lineage of mindfulness. Mindfulness, 6(1), 141-145.

Shonin, E., Van Gordon, W., \& Griffiths, M. D. (2013a). Buddhist philosophy for the treatment of problem gambling. Journal of Behavioral Addictions, 2(2), 63-71.

Shonin, E., Van Gordon, W., \& Griffiths, M. D. (2013b). Mindfulnessbased interventions: towards mindful clinical integration. Frontiers in Psychology, 4, 194.

Shonin, E., Van Gordon, W., Dunn, T. J., Singh, N. N., \& Griffiths, M. D. (2014). Meditation awareness training (MAT) for work-related wellbeing and job performance: a randomised controlled trial. International Journal of Mental Health and Addiction, 12(6), 806823.

Sik, H. H. (2010). Dharma therapy: an intervention program with mindfulness as one of its key components. In M. G. Kwee, R. T. Naylor, A. Tilakaratne, \& K. J. Gergen (Eds.), New horizons in Buddhist psychology: relational Buddhism for collaborative practitioners (pp. 353-372). Chagrin Falls: Taos Institute Publications.

Sik, H. H., \& Wu, W. Y. B. (2015). The importance of the Buddhist teaching on three kinds of knowing: in a school-based contemplative education program. In K. L. Dhammajoti (Ed.), Buddhist meditative praxis: traditional teachings \& modern applications (pp. 253-290). Hong Kong: Centre of Buddhist Studies, The University of Hong Kong.

Singh, N. N., Lancioni, G. E., Wahler, R. G., Winton, A. S., \& Singh, J. (2008). Mindfulness approaches in cognitive behavior therapy. Behavioural and Cognitive Psychotherapy, 36(06), 659-666.

Singh, N. N., Lancioni, G. E., Winton, A. S. W., Karazsia, B. T., \& Singh, J. (2013). Mindfulness training for teachers changes the behavior of their preschool students. Research in Human Development, 10(3), 211-233.

Singh, N. N., Lancioni, G. E., Winton, A. S. W., Karazsia, B. T., Myers, R. E., Latham, L. L., \& Singh, J. (2014). Mindfulness-Based Positive Behavior Support (MBPBS) for mothers of adolescents with autism spectrum disorder: effects on adolescents' behavior and parental stress. Mindfulness, 5(6), 646-657.

Singh, N. N., Lancioni, G. E., Medvedev, O. N., Myers, R. E., Chan, J., McPherson, C. L., . . Kim, E. (2018). Comparative effectiveness of caregiver training in Mindfulness-Based Positive Behavior Support (MBPBS) and positive behavior support (PBS) in a randomized controlled trial. Mindfulness. Advance of Print. 
Taylor, C., Harrison, J., Haimovitz, K., Oberle, E., Thomson, K., Schonert-Reichl, K., et al. (2016). Examining ways that a mindfulness-based intervention reduces stress in public school teachers: a mixed-methods study. Mindfulness, 7(1), $115-129$

Teasdale, J. D., Segal, Z. V., \& Williams, J. M. G. (2003). Mindfulness training and problem formulation. Clinical Psychology: Science and Practice, 10(2), 157-160.

Testing equivalence with tost in excel tutorial. (2017). Retrieved from https:/help.xlstat.com/customer/en/portal/articles/2062366-testingequivalence-with-tost-in-excel-tutorial. Accessed 16 July 2018.

Van Ginkel, J. (2014). SPSS syntax for applying rules for combining multivariate estimates in multiple imputation. Leiden: Leiden University.

Van Gordon, W., Shonin, E., Sumich, A., Sundin, E. C., \& Griffiths, M. D. (2014). Meditation awareness training (mat) for psychological well-being in a sub-clinical sample of university students: a controlled pilot study. Mindfulness, 5(4), 381-391.
Van Gordon, W., Shonin, E., \& Griffiths, M. D. (2015). Towards a second generation of mindfulness-based interventions. Australian and New Zealand Journal of Psychiatry, 49(7), 591-592.

Van Gordon, W., Shonin, E., \& Griffiths, M. D. (2016). Meditation awareness training for the treatment of sex addiction: a case study. Journal of Behavioral Addictions, 5(2), 363-372.

Van Gordon, W., Shonin, E., Dunn, T. J., Garcia-Campayo, J., \& Griffiths, M. D. (2017). Meditation awareness training for the treatment of fibromyalgia syndrome: a randomized controlled trial. British Journal of Health Psychology, 22(1), 186-206.

Virgili, M. (2015). Mindfulness-based interventions reduce psychological distress in working adults: a meta-analysis of intervention studies. Mindfulness, 6(2), 326-337.

Wallmark, E., Safarzadeh, K., Daukantaite, D., \& Maddux, R. E. (2013). Promoting altruism through meditation: an 8-week randomized controlled pilot study. Mindfulness, 4(3), 223-234.

Willams, P. (2004). Mahayana Buddhism. London, England: Routledge. 